MINI REVIEW

\title{
Intrinsic imperfections of endocrine replacement therapy
}

\author{
J A Romijn, J W A Smit and S W J Lamberts \\ Department of Endocrinology, Leiden University Medical Center, Leiden and Department of Internal Medicine, Erasmus Medical Center, \\ Rotterdam University, Rotterdam, The Netherlands \\ (Correspondence should be addressed to J A Romijn; Email: j.a.romijn@lumc.nl)
}

\begin{abstract}
Hormonal substitution therapy has been extremely successful, with respect to morbidity and mortality, in the treatment of the major syndromes of endocrine insufficiency. However, many patients treated for endocrine insufficiencies still suffer from more or less vague complaints and a decreased quality of life. It is likely that these complaints are, at least in part, caused by intrinsic imperfections of hormone replacement strategies in mimicking normal hormone secretion. Unfortunately, these complaints are often difficult to assess by clinicometric or biochemical tests, because the effects of hormones in general, and thus of hormone replacement strategies in particular, are difficult to quantify at the tissue level. Therefore, in clinical practice we rely mostly on plasma variables 'plasma endocrinology' - which are a poor reflection of hormone action at the tissue level. Appreciation of these intrinsic shortcomings of endocrine therapy is of utmost importance to prevent incorrect labelling of the complaints of many endocrine patients and to achieve further improvement in endocrine replacement strategies.
\end{abstract}

European Journal of Endocrinology 149 91-97

\section{Introduction}

In the classical concept of endocrinology, proposed by Starling in 1905, hormones are transported by the blood from endocrine glands to their site of action in distant organs. On the basis of this concept, the traditional endocrine organs were recognised and the hormones produced by these organs identified, isolated and ultimately produced in pharmaceutical quantities. In general, endocrinology has been extremely successful in the treatment of diseases caused by endocrine insufficiencies. Previously lethal diseases such as adrenal insufficiency, type 1 (insulin-dependent) diabetes mellitus and hypothyroidism, have been successfully treated for many decades. Another marker of this success is the low cost involved in the pharmaceutical production of the natural hormones. For instance, in The Netherlands, the average daily cost of thyroxine required for full thyroid replacement amounts to only $€ 0.05$, and that of hydrocortisone to only €0.10.

Although these achievements may suggest to some that endocrine research has fulfilled its promises, a perfect mimicry of endocrine homeostasis by hormone replacement therapy is in general impossible (Table 1). Fortunately, many patients receiving endocrine replacement therapy have a good quality of life. However, other patients have vague complaints and a decreased quality of life, which are difficult to assess by objective criteria. It is likely that these complaints may be explained, at least in part, by the intrinsic biological imperfections of endocrine replacement therapy. These subtle signs of endocrine imperfection may result in inadequate labelling of the complaints, if the doctor involved does not consider the possibility of imperfect endocrine replacement. Therefore, we focus here on the intrinsic imperfections of current endocrine replacement therapy.

\section{Hypothyroidism}

A minority of the patients treated with L-thyroxine for hypothyroidism have complaints despite normalisation of the euthyroid state as judged by normal concentrations of thyrotrophin (TSH). In clinical practice, these complaints are difficult to quantify by clinimetric methods or by biochemical testing. They range from musculoskeletal complaints, to vague feelings of being unwell, and to depression. Two approaches have been used to decrease the complaints experienced by these patients: an increase in the dose of L-thyroxine, and combination treatment of thyroxine with tri-iodothyronine. In some of the patients, a decrease in complaints can be achieved by increasing the dose of thyroxine above that required to restore TSH concentrations to normal (1). For this reason, such patients are often 
Table 1 Biological imperfections of endocrine therapy.

\begin{tabular}{ll}
\hline Hormone & Biological imperfections of its replacement \\
\hline DHEA & DHEA per se is probably not an active hormone, but rather a prohormone that is converted into active \\
androgens and oestrogens in target tissues by intracrine mechanisms. The influence of the dose-response \\
relationships of DHEA substitution on this intracrine regulation of hormone production is not known, although \\
DHEAS, androstenedione and testosterone concentrations increase to low normal values in females receiving \\
a daily dose of 50 mg DHEA. In addition, commercially available DHEA preparations are notoriously \\
contaminated with other hormones or substances, which in part explains why DHEA is formally not considered \\
to be a drug.
\end{tabular}

Growth hormone

Fludrocortisone

Hydrocortisone

Insulin

Testosterone

Thyroxine

Tri-iodothyronine
Although endogenous growth hormone secretion is characteristically pulsatile, the plasma profile of exogenously administered growth hormone is distinctly different. Moreover, supraphysiological concentrations of plasma growth hormone are present after administration of recombinant growth hormone for a large part of the 24-hour dose interval. Studies in rodents have documented that the molecular effects of growth hormone are dependent on the time profile of plasma hormone concentrations. At present, these effects are difficult to address in humans in vivo. In addition, markers of the biological effect of growth hormone therapy are lacking in adults, whereas assays of the surrogate marker of effect, IGF-I, are hampered by technical imperfections.

The secretion of aldosterone is pulsatile, with an alpha half-life of $\sim 30 \mathrm{~min}$. The pharmacokinetics of fludrocortisone, which is taken in a once-daily regimen, is distinctly different. Although the plasma half-life of fludrocortisone is not much different from that of aldosterone, the biological half-life is $18-36 \mathrm{~h}$. Markers of biological effect, such as serum renin/aldosterone ratio, are subject to important intra- and inter-assay variabilities.

Plasma profiles of exogenously administered hormone are distinctly different from normal pulsatile profiles. Moreover, before the morning dose of hydrocortisone is taken, extremely low concentrations of cortisol may be found, which is not unexpected in view of the twice-daily dose interval and the plasma half-life of $\sim 80 \mathrm{~min}$. As is the case for growth hormone, adequate markers of biological effect are lacking entirely, leading to crude dosing schedules related to body mass index, and potential over- or undertreatment.

The half-life of endogenous insulin is several minutes, whereas that of short-acting exogenous insulin, administered s.c., is several hours. The plasma profile of s.c.-administered insulin is distinctly different from that of endogenously secreted insulin. A first-phase response can not be mimicked. In addition, exogenous insulin lacks the pulsatile characteristics of endogenous insulin secretion. In vivo secretion of insulin in humans during glucose stimulation consists of a series of distinct secretory bursts, which accounts for the major part of insulin secretion. Of all the hormone therapies considered in this article, the artificial administration of insulin is the one most clearly reflected in inadequate pharmacodynamics, when one takes into account the narrow therapeutic window between insulin profiles and the ultimate goal of euglycaemia.

Endogenously produced testosterone has a very short half-life, and consequently modified testosterone derivatives with longer half-lives are used. Endogenous testosterone is produced in a pulsatile fashion, which is absent in testosterone therapy. Markers of biological effect are subjective or extrapolated from highly variable gonadotrophin concentrations in primary hypogonadism.

The thyroid secretes tri-iodothyronine $\left(T_{3}\right)(\sim 20 \%)$ in addition to thyroxine $\left(T_{4}\right)(\sim 80 \%)$. In the absence of thyroid function, exogenous thyroxine is not able to normalise the concentrations of $T_{4}$ and $T_{3}$ in all tissues in rodents, even in the presence of normal TSH concentrations. Despite this knowledge, currently available preparations of $\mathrm{T}_{3}$ have unfavourable pharmacological profiles and adequate markers of biological effect are lacking. Additional evidence is required before combination therapy can be advised.

There are no slow-release preparations of $T_{3}$, which would provide stable plasma concentrations in view of the half-life of $T_{3}\left(\sim 1\right.$ day). The optimal dose is uncertain. The relationship between plasma concentrations of $T_{3}$ and tissue-specific concentrations of $T_{3}$ in humans is unknown during $T_{3}$ therapy in hypothyroidism. allowed to take a dose of thyroxine that would be judged as overtreatment with respect to TSH concentrations. The second approach was evaluated by Bunevicius et al. (2), who performed a randomised controlled trial to compare the effects of thyroxine alone with those of thyroxine plus tri-iodothyronine. Patients with hypothyroidism benefitted when $12.5 \mu \mathrm{g}$ triiodothyronine was substituted for $50 \mu \mathrm{g}$ thyroxine in their treatment regimens. This resulted in improved neuropsychological functioning. Pulse rates and serum sex hormone-binding globulin concentrations were greater after treatment with thyroxine plus tri-iodothyronine, indicating a slightly greater effect on the heart and liver. Serum thyroxine concentrations were lower and tri-iodothyronine concentrations were greater after treatment with thyroxine plus tri-iodothyronine, but serum TSH concentrations, a sensitive measure of thyroid hormone action, were similar after the two treatments. It should be noted that not all patients benefitted from this approach because, even in the group with combination therapy, patients continued to report complaints of depression.

In clinical practice, the adequacy of thyroid hormone supplementation is assessed by the measurement of 
TSH concentrations. This approach deserves two comments. First, it is remarkable that the normal values of TSH show a more than tenfold variation, between 0.4 and $4.5 \mathrm{mU} / \mathrm{l}$. Because, in clinical practice, the optimal TSH concentration for individual patients within this range is unknown, titration of the substitution dose of thyroxine within this tenfold variation is relatively crude. Secondly, the intrinsic assumption of many doctors in this approach is that normal TSH concentrations reflect adequate thyroid hormone concentrations, not only at the tissue level of the hypothalamus and the pituitary, but also in the other tissues. However, it is likely that this assumption is erroneous, because TSH is produced only by the pituitary gland and therefore may not reflect thyroid hormone status in tissues outside the hypothalamopituitary axis. This notion is supported by data obtained from animal experiments. The thyroid secretes predominantly thyroxine, and only a small amount of tri-iodothyronine. Thyroxine is considered to be an inactive hormone, because a thyroxine-specific receptor has not been identified. Rather, thyroxine serves as a prohormone, because it is the precursor of tri-iodothyronine. The availability of tri-iodothyronine in target tissues is regulated by tissue-specific deiodinases. Some tissues, such as muscle, have a relatively low deiodinase activity and are dependent, to a great extent, on tri-iodothyronine derived from the thyroid and the liver. Other tissues, such as the brain and liver, have a high deiodinase activity and, as a consequence, the availability of tri-iodothyronine within those tissues is determined within the tissues themselves.

In rodents, it has been clearly demonstrated that there is no single dose of thyroxine or tri-iodothyronine that normalises thyroid hormone concentrations in all tissues simultaneously in hypothyroid animals (3). Therefore, it is highly likely that, in patients treated with L-thyroxine, subtle derangements at the tissue level are present with respect to thyroid hormone availability, and probably also thyroid hormone action. Unfortunately, we lack sensitive signs and symptoms to evaluate this in clinical practice. In addition, we do not have sensitive biochemical markers of thyroid hormone action at the tissue level other than TSH.

\section{Growth hormone deficiency}

Different pituitary disorders cause growth hormone deficiency. In children this results in growth retardation. In adults, the term 'growth hormone' is a misnomer, as it suggests that growth hormone does not serve a function in individuals with closed epiphysial plates. Growth hormone became available in pharmaceutical amounts through recombinant technology in the 1980s. Since then, a large number of studies of growth replacement therapy have shown objective and subjective improvements in a large number of parameters in adults who are growth hormone-deficient. This has resulted in the inclusion of growth hormone in the standard treatment for adults with growth hormone deficiency. Nonetheless, there are intrinsic imperfections of growth hormone therapy. In healthy individuals, growth hormone is secreted in large bursts, especially during sleep. This pulsatile pattern of secretion is important for growth hormone to be effective. For instance, in a recent study by Tannenbaum et al. (4), the effects of growth hormone on the liver were not constant, but dependent on the time profile of growth hormone secretion. In growth hormone-deficient individuals, however, growth hormone is injected s.c. once daily. After injection, the drug is released slowly from its subcutaneous pool, resulting in slowly increasing and subsequently declining plasma concentrations. This pharmacokinetic profile is distinctly different from the endogenous, pulsatile pattern of secretion.

Another problem is the establishment of an optimal dose of growth hormone. Because there are no sensitive signs and symptoms with which to assess the adequacy of growth hormone substitution in individual patients, we rely on the measurement of insulin-like growth factor (IGF)-I as an integrative marker of growth hormone availability. However, IGF-I assay is one of the most problematic endocrine assays, because of large inter- and intra-assay variations: it is not uncommon to have coefficients of variation of $15-20 \%$. As a consequence, the assays may report a value of $20 \mathrm{nmol} / \mathrm{l}$, anywhere in the range between 14 and $28 \mathrm{nmol} / \mathrm{l}$. In clinical trials, this analytical variation in the measurement of IGF-I can be decreased by increasing the number of measurements at each time point, by optimising the assays and by analysis of the samples from each individual in the same run. However, this is impractical in clinical practice and therefore it is almost impossible to assess the exact, optimal dose of growth hormone in growth hormone-deficient patients.

\section{Type 1 diabetes mellitus}

Type 1 diabetes mellitus is caused by absolute insulin deficiency resulting from $\beta$-cell destruction. Treatment of type 1 diabetes mellitus has been available since the discovery of insulin by Banting \& Best in 1922 . Subsequently, major improvements in pharmaceutical production have been achieved. Continuing development of 'designer' insulins with better pharmacokinetic profiles is enabling a better mimicking of normal insulin secretory profiles. The standard of care is aimed at achieving normoglycaemia through intensive insulin treatment, because this results in a major reduction of chronic complications, as shown by the Diabetes Control and Complications Trial (DCCT) (5). Nonetheless, even with intensive insulin treatment, a 
considerable proportion of the patients develop long-term complications, and such treatment is thus not perfect. Moreover, there is a delicate balance between the optimal insulin regimens to prevent hyperglycaemia and the induction of hypoglycaemia. In the DCCT, the incidence of symptomatic hypoglycaemia was increased two- to threefold in the intensively treated group as compared with the control group (6). However, the DCCT may have been biased in this respect, because patients with hypoglycaemia unawareness were excluded from this trial. Continuous monitoring with subcutaneous glucosensors indicated that patients with type 1 diabetes mellitus have frequent episodes of both hyper- and hypoglycaemic episodes that are not evident with routine testing (7). Therefore, the dysregulation of glucose metabolism in intensively treated patients with type 1 diabetes mellitus is probably worse than hitherto recognised.

From the perspective of monitoring endocrine substitution therapy, type 1 diabetes mellitus is a very interesting disease. In contrast to all other hormonal substitutions, insulin treatment of type 1 diabetes mellitus can be monitored by a perfect marker of hormone action at the tissue level: plasma glucose concentrations. Moreover, plasma glucose represents an integrative marker of insulin action, because it represents the balance between glucose production and glucose uptake. However, type 1 diabetes mellitus is also a prime example demonstrating that perfect endocrine substitution is in general very difficult, despite this availability of frequent glucose measurements as a marker of tissue hormone action. This provides food for thought for the imperfection of hormonal replacement strategies in other endocrine deficiencies, for which we lack ideal markers of hormone action at the tissue level that are comparable to plasma glucose.

\section{Adrenal insufficiency}

\section{Hydrocortisone}

The introduction of glucocorticoids in the middle of the last century has revolutionised the treatment of adrenal insufficiency, a previously lethal disease. Replacement therapy requires $20-30 \mathrm{mg}$ hydrocortisone divided into two or three doses a day. Alternatively, longeracting corticosteroid preparations are used, such as dexamethasone $0.5 \mathrm{mg}$ /day or prednisone $5 \mathrm{mg}$ /day. The notion proposed by the proponents of these longer-acting equivalents is that these alternative approaches avoid the high peak concentrations and periods of inadequate replacement that are associated with the use of hydrocortisone.

It is advised that replacement therapy for patients with adrenal insufficiency should be individualised. Parameters for judging the adequacy of glucocorticoid substitution involve blood pressure, heart rate, sense of wellbeing, and elimination of symptoms such as anorexia, nausea and vomiting. In addition, signs of excess glucocorticoids may dictate a decrease in dose. In our experience, however, slight changes in the dose of hydrocortisone within the range $20-35 \mathrm{mg} /$ day are hardly, if at all, reflected in changes in these parameters. Nonetheless, long-term replacement therapy with hydrocortisone in a dose of $30 \mathrm{mg} /$ day is associated with decreased bone mineral density (8). In addition, measurements of rates of production of cortisol using stable isotopes in vivo have indicated that the physiological rate of production of cortisol was much lower than previously believed: $5.7 \mathrm{mg} / \mathrm{m}^{2}$ per day compared with $12-15 \mathrm{mg} / \mathrm{m}^{2}$ per day (9). Therefore, the currently recommended dose of hydrocortisone is lower than before. Thus, on the basis of the signs and symptoms, it is apparently impossible to determine the perfect dose of hydrocortisone for physiological replacement in individual patients. Moreover, a reduction from the previously used, greater doses of hydrocortisone to the currently recommended dose did not result in any detectable change in clinical parameters in individual patients. Finally, we lack reliable biochemical markers of glucocorticoid action at the tissue level. This is because there is not a single physiological function that is controlled by cortisol alone.

From these arguments, we conclude that adjustment of the perfect dose of hydrocortisone for individual patients with adrenal insufficiency is not possible. In addition, our advice to patients to adapt their therapeutic hydrocortisone regimen in the case of stress or intercurrent disease is largely based on intuition, rather than on hard experimental data (10).

\section{Fludrocortisone}

Patients with primary adrenal insufficiency also have aldosterone deficiency, the signs and symptoms of which are limited to postural hypotension. Mineralocorticoid replacement with fludrocortisone is obligatory in these patients, the recommended daily dose of the fludrocortisone ranging between 0.05 and $0.20 \mathrm{mg}$. The adequacy of mineralocorticoid treatment is assessed by measuring the postural blood pressure and serum sodium, potassium and renin concentrations. Plasma renin correlates better with mineralocorticoid dose than do plasma sodium and potassium concentrations (11). However, even optimal treatment with fludrocortisone may be associated with mildly increased plasma renin activity. The measurement of serum potassium is useful to assess asymptomatic overdosage of fludrocortisone, which is indicated by low potassium values, rather than by plasma renin activity (12). These observations on the relationship between dose of fludrocortisone, plasma renin and electrolyte concentrations were mostly made in carefully balanced studies, which are impractical in clinical practice. Therefore, most doctors rely only on postural blood 
pressure measurements and serum sodium and potassium concentrations. These variables remain within the normal range in most patients with primary adrenal insufficiency who are receiving the above-mentioned doses of fludrocortisone. Consequently, the choice for a dose of fludrocortisone within the indicated range is based on relatively crude criteria.

\section{Dehydroepiandrosterone (DHEA)}

Adrenal insufficiency requires life-long replacement therapy with glucocorticoids; in primary adrenal insufficiency, life-long replacement therapy with mineralocorticoids is also necessary. In addition, however, the adrenals produce DHEA and dehydroepiandrosterone sulphate (DHEAS), and adrenal insufficiency is associated with decreased plasma concentrations of these steroids. In peripheral tissues such as hair follicles, adipose tissue and prostate, DHEA and DHEAS are converted into active androgens such as androstenedione, testosterone and dihydrotestosterone, and into active oestrogens such as oestrone and oestradiol.

Several trials have evaluated the effect of DHEA replacement in primary and secondary adrenal insufficiency. DHEA per se is probably not an active hormone, because a receptor for DHEA has not been identified. Rather, DHEA and DHEAS serve as precursors of steroid biosynthesis within other tissues and act after conversion into androgens and oestrogens, by activating androgen and oestrogen receptors. In response to treatment with DHEA $50 \mathrm{mg} /$ day by mouth, concentrations of DHEAS, androstenedione and testosterone increase to low-normal values in females. The results of randomised, double-blind controlled trials indicate that DHEA improves mood, wellbeing and, in women, libido, in the absence of significant short-term side effects $(13,14)$. In clinical practice this has resulted in the introduction of DHEA treatment in adrenal insufficiency, in the above-mentioned dose. However, monitoring of this replacement strategy by signs and symptoms is not really possible in clinical practice.

\section{Hypogonadism}

\section{Men}

Androgens have been used for the treatment of male hypogonadism for more than 60 years. It has been well documented that this replacement therapy results in reversal of the effects of hypogonadism with improvement of sexual function, mood, bone mineral density and lean body mass. However, clinical signs and symptoms are not sufficiently sensitive to enable titration of the dose of testosterone derivatives. For instance, the improvement of sexual function and mood variables by testosterone treatment requires only a minimally adequate concentration of testosterone and further increases in plasma concentrations do not confer further improvement in mood variables (15). In contrast, it has been well established that pharmacological dosages of androgens result in further increase in lean body mass.

In addition to the lack of sensitive signs and symptoms for monitoring the adequacy of testosterone substitution, clinicians have not achieved complete success in mimicking normal testosterone homeostasis. Because testosterone per se is degraded rapidly, it is necessary to modify the molecule in order to alter its metabolism and thereby increase the plasma half-life. Testosterone derivatives have been developed for oral, parenteral and transdermal administration. Because orally administered testosterone is subject to a major first-pass effect through the liver, $17 \alpha$-alkylated derivatives are used for oral substitution. However, plasma testosterone concentrations cannot be used to monitor substitution with these compounds. In addition, these drugs are inferior with respect to their efficacy and are therefore not the drugs of first choice. A number of testosterone derivatives are available for parenteral use. The limitations of these depot preparations are fluctuating testosterone concentrations, resulting in initially high concentrations and insufficient concentrations before the next dose. Transdermal preparations appear to mimic physiological testosterone concentrations, even with a diurnal variation. However, there are indications that even transdermal testosterone does not result in a complete mimicking of normal testosterone secretion. For instance, gonadotropin concentrations remain increased in a large proportion of the patients, despite plasma concentrations of testosterone, dihydrotestosterone and oestradiol within the normal range (16). Consequently, androgen replacement therapy by testosterone derivatives is not perfect, but is subject to intrinsic imperfections.

\section{Women}

Treatment of primary and secondary hypogonadism in women consists of administration of oestrogens and, if a uterus is present, progestins. Oestrogen supplementation reverses the effects of hypogonadism in women, including those who are postmenopausal. It appears to be difficult to evaluate the adequacy of oestrogen supplementation in hypogonadism by clinicometric approaches. The effects of oestrogens on the skin, mood, vaginal moisturisation etc. are undisputed, but lack clear dose-response relationships. In biochemical evaluations, there are no specific tissue markers of oestrogen activity that can be used in individual patients, apart from luteinising hormone (LH) and follicle-stimulating hormone. Moreover, oestrogen metabolism is very complicated and oestrogen supplementation does not completely mimic the kinetics and metabolism of endogenous oestrogen. 
There have been only a few trials of the effects and complications of oestrogen therapy in primary and secondary hypogonadism in women of premenopausal age. Nonetheless, in this respect useful information can be deduced from large-scale trials that have been conducted on the effects and complications of oestrogens used for contraception and for postmenopausal hormone replacement. In these trials, the dose regimens of oestrogens were fixed, rather than titrated in individualised patients. The dose of oestrogens in oral contraceptives is derived from analysis of the balance between the efficacy in preventing pregnancy and the adverse effects, rather than from the perspective of physiological rates of production of oestradiol. These studies on oral contraception reflect the intrinsic problems in the assessment of the effects of oestrogen supplementation in individual patients: within this range of oestrogen dosages there are no signs, symptoms or biochemical markers of effects of the hormones at the tissue level. Apparently, there is no linear dose-response relationship for oestrogens that can be used in clinical practice. Similar remarks can be made with respect to postmenopausal hormone replacement. Clinical and biochemical parameters that can be used in clinical practice to assess the adequacy of oestrogen treatment are limited. This is also reflected in the necessity to perform huge trials in order to establish whether nature has failed women by depriving them of oestrogens for one-third of their life.

\section{Hypoparathyroidism}

Because, at present, parathyroid hormone is not available for the treatment of hypoparathyroidism, the standard treatment of this endocrine deficiency syndrome consists of calcium and vitamin D analogues. Although serum calcium concentrations can be restored to normal by this approach, calcium/ phosphate homeostasis is clearly altered in these patients; clinical symptoms that they may exhibit include nephrolithiasis (17). In addition, calcium and vitamin D analogues fail to restore wellbeing in hypoparathyroidism, reflected in increased scores for anxiety, phobic anxiety and their physical equivalents (17).

\section{Implications of genetic variations in hormonal (receptor) genes}

There is increasing evidence that there are genetically determined differences in hormone secretion and hormone sensitivity. For instance, early studies into the relationships between serum TSH and free thyroxine concentrations have indicated that a certain reproducible, genetically determined individual set-point exists in normal persons at which the feedback control of the pituitary-thyroid axis is set (18). In addition, glucocorticoid sensitivity, as measured by the dexamethasone-mediated suppression of early morning cortisol concentrations, is quite variable between, but highly reproducible within, individuals (19). Interestingly, a number of frequently occurring polymorphisms in and around exon 2 of the glucocorticoid receptor gene are associated with an increased $(20,21)$ or decreased (22) sensitivity to glucocorticoids. Presence of a high number of CAG repeats within the androgen receptor gene attenuates the effects of testosterone on bone density and bone metabolism (23), whereas a decreased number of CA repeats in the IGF-I gene is associated with a lower birth weight, lower body height and serum IGF-I concentrations at the lowest quartile of normal $(24,25)$. A polymorphism in the LH gene and the ensuing variant serum concentrations of LH reflect androgen bioactivity in elderly men in a different manner than in individuals with wild-type LH (26). Oestrogen receptor polymorphisms considerably modify the effects of hormone replacement therapy on concentrations of high-density lipoprotein cholesterol and other outcomes related to oestrogen treatment in postmenopausal women (27).

These recently obtained new insights into the genetic variations (polymorphisms) of hormones and their receptors have provided important new insights into the individual set-points of activity of the hormonal axes involved, and into the mechanisms of the respective differences of hormone sensitivity at the level of the target tissues. The challenge of clinical endocrinology in the coming years will be to apply this knowledge to the treatment of individual patients in order to optimize the dose and frequency of hormone replacement.

\section{Conclusion}

Hormonal substitution therapy has been very successful in treating the major syndromes of endocrine insufficiency with respect to mortality. However, endocrine substitution does not reproduce the normal plasma hormone profiles of healthy individuals. Moreover, the effects of hormones in general are difficult to quantify at the tissue level. Consequently, titration of endocrine replacement therapy is possible only within certain limits. It is very likely that these intrinsic imperfections in endocrine replacement therapies result in subtle physiological derangements. Most importantly, this imperfection in endocrine substitution therapy may result in impaired quality of life, which can not easily be assessed by objective criteria in clinical practice. Recognition of these intrinsic shortcomings of endocrine treatment is of utmost importance for appreciation of the complaints of some of these patients and to prevent incorrect labelling of these complaints. 


\section{References}

1 Carr D, McLeod DT, Parry G \& Thornes HM. Fine adjustment of thyroxine replacement dosage: comparison of the thyrotrophin releasing hormone test using a sensitive thyrotrophin assay with measurement of free thyroid hormones and clinical assessment. Clinical Endocrinology 198828 325-333.

2 Bunevicius R, Kazanavicius G, Zalinkevicius R \& Prange AJJ. Effects of thyroxine as compared with thyroxine plus triiodothyronine in patients with hypothyroidism. New England Journal of Medicine 1999340 424-429.

3 Escobar-Morreale HF, del Rey FE, Obregon MJ \& de Escobar GM. Only the combined treatment with thyroxine and triiodothyronine ensures euthyroidism in all tissues of the thyroidectomized rat. Endocrinology 1996137 2490-2502.

4 Tannenbaum GS, Choi HK, Gurd W \& Waxman DJ. Temporal relationship between the sexually dimorphic spontaneous GH secretory profiles and hepatic STAT5 activity. Endocrinology $20011424599-4606$.

5 The Diabetes Control and Complications Trial Research Group. The effect of intensive treatment of diabetes on the development and progression of long-term complications in insulin-dependent diabetes mellitus. New England Journal of Medicine 1993329 977-986.

6 The Diabetes Control and Complications Trial Research Group. Hypoglycemia in the Diabetes Control and Complications Trial. Diabetes 199746 271-286.

7 Boland E, Monsod T, Delucia M, Brandt CA, Fernando S \& Tamborlane WV. Limitations of conventional methods of selfmonitoring of blood glucose: lessons learned from 3 days of continuous glucose sensing in pediatric patients with type 1 diabetes. Diabetes Care 200124 1858-1862.

8 Zelissen PM, Croughs RJ, van Rijk PP \& Raymakers JA. Effect of glucocorticoid replacement therapy on bone mineral density in patients with Addison disease. Annals of Internal Medicine 1994 $120207-210$.

9 Esteban NV, Loughlin T, Yergey AL, Zawadzki JK, Booth JD, Winterer JC et al. Daily cortisol production rate in man determined by stable isotope dilution/mass spectrometry. Journal of Clinical Endocrinology and Metabolism 1991 72 39-45.

10 Lamberts SW, Bruining HA \& de Jong FH. Corticosteroid therapy in severe illness. New England Journal of Medicine 1997337 $1285-1292$.

11 Oelkers W, Diederich S \& Bahr V. Diagnosis and therapy surveillance in Addison's disease: rapid adrenocorticotropin (ACTH) test and measurement of plasma ACTH, renin activity, and aldosterone. Journal of Clinical Endocrinology and Metabolism $199275259-264$.

12 Thompson DG, Mason AS \& Goodwin FJ. Mineralocorticoid replacement in Addison's disease. Clinical Endocrinology 197910 499-506.

13 Arlt W, Callies F, van Vlijmen JC, Koehler I, Reincke M, Bidlingmaier $\mathrm{M}$ et al. Dehydroepiandrosterone replacement in women with adrenal insufficiency. New England Journal of Medicine 1999341 1013-1020.

14 Hunt PJ, Gurnell EM, Huppert FA, Richards C, Prevost AT, Wass JA et al. Improvement in mood and fatigue after dehydroepiandrosterone replacement in Addison's disease in a randomized, double blind trial. Journal of Clinical Endocrinology and Metabolism 200085 4650-4656.

15 Wang C, Alexander G, Berman N, Salehian B, Davidson T, McDonald $\mathrm{V}$ et al. Testosterone replacement therapy improves mood in hypogonadal men - a clinical research center study. Journal of Clinical Endocrinology and Metabolism $1996 \mathbf{8 1}$ $3578-3583$.
16 Dobs AS, Meikle AW, Arver S, Sanders SW, Caramelli KE \& Mazer NA. Pharmacokinetics, efficacy, and safety of a permeation-enhanced testosterone transdermal system in comparison with bi-weekly injections of testosterone enanthate for the treatment of hypogonadal men. Journal of Clinical Endocrinology and Metabolism 199984 3469-3478.

17 Arlt W, Fremerey C, Callies F, Reincke M, Schneider P, Timmermann Wet al. Well-being, mood and calcium homeostasis in patients with hypoparathyroidism receiving standard treatment with calcium and vitamin D. European Journal of Endocrinology 2002146 215-222.

18 Spencer CA, LoPresti JS, Patel A, Guttler RB, Eigen A, Shen D et al. Applications of a new chemiluminometric thyrotropin assay to subnormal measurement. Journal of Clinical Endocrinology and Metabolism $1990 \mathbf{7 0} 453-460$.

19 Huizenga NA, Koper JW, de Lange P, Pols HA, Stolk RP, Grobbee DE et al. Interperson variability but intraperson stability of baseline plasma cortisol concentrations, and its relation to feedback sensitivity of the hypothalamo-pituitary-adrenal axis to a low dose of dexamethasone in elderly individuals. Journal of Clinical Endocrinology and Metabolism 199883 47-54.

20 Huizenga NA, Koper JW, De Lange P, Pols HA, Stolk RP, Burger H et al. A polymorphism in the glucocorticoid receptor gene may be associated with an increased sensitivity to glucocorticoids in vivo. Journal of Clinical Endocrinology and Metabolism $1998 \mathbf{8 3}$ 144-151.

21 van Rossum EF, Huizenga NA, Uitterlinden AG, Janssen JA, Brinkmann AO, de Jong $\mathrm{FH}$ et al. Identification of a restriction site polymorphism in the glucocorticoid receptor gene: association with increased sensitivity to glucocorticoids in vivo, corticosteroid-binding globulin and systolic blood pressure. 84th Annual Meeting of the Endocrine Society, San Francisco 2002 OR-49-41.

22 van Rossum EF, Koper JW, Uitterlinden AG, Huizenga NA, Janssen JA, Brinkmann, AO et al. A polymorphism in the glucocorticoid receptor gene, which decreases sensitivity to glucocorticoids in vivo, is associated with a healthier metabolic profile. 84th Annual Meeting of the Endocrine Society, San Francisco 2002 P3-334.

23 Zitzmann M, Brune M, Kornmann B, Gromoll J, Junker R \& Nieschlag E. The CAG repeat polymorphism in the androgen receptor gene affects bone density and bone metabolism in healthy males. Clinical Endocrinology 200155 649-657.

24 Vaessen N, Heutink P, Janssen JA, Witteman JC, Testers L, Hofman A et al. A polymorphism in the gene for IGF-I: functional properties and risk for type 2 diabetes and myocardial infarction. Diabetes $200150637-642$.

25 Vaessen N, Janssen JA, Heutink P, Hofman A, Lamberts SW, Oostra BA et al. Association between genetic variation in the gene for insulin-like growth factor-I and low birthweight. Lancet 2002359 1036-1037.

26 van den Beld A. Huhtaniemi IT, Pettersson KS, Pols HA, Grobbee $\mathrm{DE}$, de Jong $\mathrm{FH}$ et al. Luteinizing hormone and different genetic variants, as indicators of frailty in healthy elderly men. Journal of Clinical Endocrinology and Metabolism 199984 1334-1339.

27 Herrington DM, Howard TD, Hawkins GA, Reboussin DM, Xu J, Zheng SL et al. Estrogen-receptor polymorphisms and effects of estrogen replacement on high-density lipoprotein cholesterol in women with coronary disease. New England Journal of Medicine 2002346 967-974.

Received 19 March 2003

Accepted 25 March 2003 